\title{
Cognitive Studies for Cancer Survivors in India: Is This the Right Time or Should we Cross the Bridge only When we will Come to it?
}

\section{Introduction and Rationale}

Cancer is gradually emerging as leading cause of deaths in India with more than 2.5 million patients suffering from the disease. ${ }^{[1]}$ This increase in incidence can either be attributed to increased lifespan and better diagnostic techniques or it may reflect a realistic pattern due to exposure to risk factors for cancer.

India is still grappling with overwhelmingly poor outcomes in cancer patients due to a variety of reasons including late stages at presentation, delay in diagnosis due to poor referral system, capricious public health-care system and infrastructure, high abandonment rates, and lack of availability of newer drugs, whereas developed world is now focusing on perspectives such as de-escalation of therapies, preservation of fertility, cardiotoxicity, and cognitive issues that help cancer survivors getting better quality of life (qol) and living a more productive life. Through this commentary, we have focused on the aspect of cognition related to chemotherapy or cancer per se and its relevance to India. Besides, we have also proposed the schema of studies that need to be conducted and challenges ahead.

Cancer-induced cognitive dysfunction encompasses range of difficulties that tend to include relatively subtle changes in memory, concentration, and executive function that can show up in the weeks during and months after completion of cancer treatment and persist thereafter in up to $75 \%$ of cancer patients. ${ }^{[2]}$

The etiology of cognitive deficits in cancer patients could be multifactorial (psychological stress, occurrence of cancer itself, brain irradiation, intrathecal chemotherapy, direct treatment for brain cancer or metastasis, and systemic chemotherapy). Its importance lies in the significant distress in professional reintegration, interpersonal relationships, and leisure activities. This might also affect future qol and might bring a feeling of worthlessness.

\section{Contemporary Evidence: Clinical}

Among the common tumors and in tumors in which relatively longer outcomes are expected, cognitive deficit is found in $20 \%-40 \%$ patients as per various studies. ${ }^{[3]}$ Cruzado et al. in a longitudinal study of FOLFOX 4 chemotherapy in colorectal patients found mild and transient negative effect of chemotherapy on verbal memory. Surprisingly, in this study, $37 \%$ of patients had cognitive impairment in the prechemotherapy evaluation giving emphasis on the proposition that cancer per se might cause cognitive dysfunction. ${ }^{[4]}$ Similarly, in a series of lung cancer patients, Simó et al. demonstrated cognitive impairment before and after chemotherapy. Before starting the treatment, patients showed verbal memory deficits and widespread white matter damage. ${ }^{[5]}$ This underscores the fact that cancer patients have preexisting cognitive dysfunction and could be hypothesized to be related to interactions between inherent metabolic activity of cancer, systemic inflammation by cytokines, and brain function. Thus, the baseline cognitive status (prechemotherapy) is essential so as to ascertain the exact cognitive deficit. ${ }^{[6]}$

The occurrence of cognitive dysfunction has been replicated in variety of malignancies. In a recent study, neuropsychological assessments were done to determine adjuvant chemotherapy's association with cognitive dysfunction in men with nonseminomatous germ cell tumors. The authors found significantly high risk of cognitive dysfunction at 12 months of therapy in chemotherapy as compared to surveillance group in dose-dependent manner. ${ }^{[7]}$ Further, Jacola et al documented that attention deficits were more pronounced with higher intensity chemotherapy in survivors of acute lymphoblastic lymphoma. ${ }^{[8]}$ It can be inferred that dose/intensity and duration of treatment can affect cognition as a direct correlate.

With these reinforcing data, it seems that cognition is a real problem and needs to be addressed at least in cancers with good long-term outcomes.

\section{Contemporary Evidence: Functional magnetic resonance imaging and electroencephalography}

Majority of the studies conducted till date have used standard neuropsychological tests to assess cognitive deficits, pertaining to memory, attention, concentration, learning, executive functions, and processing speed. However, magnitude of these deficits both in terms of structural changes, leading to functional manifestations, has been less addressed till now. Electroimaging (electroencephalography [EEG]) and neuroimaging (functional magnetic resonance imaging [fMRI]) could serve to evaluate the cognitive deficits and to follow up in these patients.

Several brain imaging-related studies have reported that there is a decrease in integrity of white matter, which correlates with processing speed. Inagaki et al. studied regional brain volume difference in breast cancer patients exposed to chemotherapy with those not exposed using MRI. ${ }^{[9]}$ They reported reduced superior frontal gyri and parahippocampal gyrus volume, which correlated 
with poor attention/concentration and impaired memory performance, respectively, 4 months after the completion of chemotherapy, though no change was observed 1-year posttreatment. Recently, Jenkins et al. found reduction in the volume of subgenual, dorsal anterior cingulate and inferior temporal gyrus in chemotherapy group. ${ }^{[10]}$ Conflicting reports are available for fMRI studies in terms of hypoactivation and hyperactivation in brain activities when breast cancer patients on chemotherapy were compared to controls. ${ }^{[11]}$

Recently, Bruno et al. in 2012 studied resting state networks in patients of breast cancer as compared to healthy controls using fMRI. They found that there is disrupted default mode network in frontal, striatal, and temporal regions of the brain. ${ }^{[12]}$

Quantitative EEG can be used as a better tool not only for assessing connectivity but also can decipher temporal dynamics. Only one pilot study has been done so far by Moore et al. in 2014 using quantitative EEG on 16 electrode data. They studied that EEG power measurement has the potential to provide a sensitive neurophysiologic correlate of cancer treatment-related fatigue and cognitive dysfunction in seven pairs of case-control breast cancer patients and found that EEG amplitude was increased in breast cancer patients as compared to controls. ${ }^{[13]}$ Further, source estimation was not done in these patients. Quantitative EEG (128 electrodes) will be an apt tool to address wide gap in the literature. It has been shown that the application of more than 100 EEG channels is not only favorable but also necessary to guarantee a reasonable accuracy in the calculations of cortical source density or cortical mapping. ${ }^{[14]}$ There are experimental evidence supporting that denser EEG systems can help recover more information. ${ }^{[15]}$ Brodbeck et al. showed that epileptic focus localization improved (sensitivity and specificity) using 128 electrode arrays as compared to 32 channel arrays. ${ }^{[16]}$

Thus, using 128 channels, EEG improves localization, and as compared to MRI, it has superlative temporal resolution as well. ${ }^{[17]}$

Mathan et al. further studied efficacy of EEG-based estimation of cognitive load among individuals experiencing cancer-related cognitive deficits. However, the effect of chemotherapy on cognition was not documented. ${ }^{[18]}$ Pilot study results regarding the feasibility of electroencephalography biofeedback to reduce subjective cognitive complaints for breast cancer survivors also demonstrated significant improvements in perceived cognitive function $(n=23) .{ }^{[19]}$ Thus, these modalities can be used for intervention, thereby improving prognosis in these patients.

\section{Indian Data and its Implications}

Unfortunately, if we dwell upon Indian statistics, there seems to be negligible research in this area. Sequeira et al. in a case-control study of 60 patients comparing 30 patients with breast cancer revealed a significant cognitive deficit in patients as compared to controls. ${ }^{[1]}$ It is imperative to prioritize not only diagnosis and treatment but also improving secondary outcomes such as cognition. This may be all the more important in young patients, wherein cognitive deficit may ruin their qol both personally and professionally. The impact of cancer and cancer treatment on health-care costs is not only due to direct cost incurring due to treatment but also due to indirect costs pertaining to loss of days from work both during and after treatment (due to iatrogenic causes). Moreover, the intangible cost like suffering for both the patient and the caregiver are difficult to measure.

Thus, onco-economics does cause a significant burden on nation and individual (especially in country like India where there is a lack/limited health insurance). Addressing them at the appropriate time may help them in decision-making process during chemotherapy and significantly improve their qol.

\section{What next?}

The question arises as to what strategies must be undertaken to bridge the gap between what is done and what needs to be done so as to improve both diagnosis and prognosis of the patient during and after therapeutic interventions. First of all, awareness about cognitive effects to both health providers and patients/caregivers so that appropriate remedial measures may be taken to improve the outcome. Second, task force may be formed to address the secondary outcomes of cancer survivors. Last but not least, funding/ grants to investigators to carry out research addressing secondary outcomes may work hand in hand to complement conventional diagnosis and treatment.

Such strategies have the potential of improving cognitive deficits due to chemotherapy/cancer and may secondarily provide more satisfaction and better onco-economics in cancer survivors.

Keeping this in mind, we aim to initiate our research on cognition and cancer and we welcome collaborators who share similar vision.

Financial support and sponsorship

Nil.

\section{Conflicts of interest}

There are no conflicts of interest.

\section{Sameer Rastogi, Ratna Sharma ${ }^{1}$, Simran Kaur ${ }^{1}$}

Departments of Medical Oncology and 'Physiology, All India Institute of Medical Sciences, New Delhi, India

Address for correspondence: Dr. Simran Kaur, Department of Physiology, All India Institute of Medical Sciences,

New Delhi, India.

E-mail: simranaiims@outlook.com 


\section{References}

1. Sequeira AK, Sequeira AZ, Krishnamurthy K. Study of cognitive functions in breast cancer patients: A case control study. Muller J Med Sci Res 2014;5:129-33.

2. Jean-Pierre P. Management of cancer-related cognitive dysfunction-conceptualization challenges and implications for clinical research and practice. US Oncol 2010;6:9-12.

3. Jansen CE, Miaskowski C, Dodd M, Dowling G, Kramer J. A metaanalysis of studies of the effects of cancer chemotherapy on various domains of cognitive function. Cancer 2005;104:2222-33.

4. Cruzado JA, López-Santiago S, Martínez-Marín V, José-Moreno G, Custodio AB, Feliu J, et al. Longitudinal study of cognitive dysfunctions induced by adjuvant chemotherapy in colon cancer patients. Support Care Cancer 2014;22:1815-23.

5. Simó M, Root JC, Vaquero L, Ripollés P, Jové J, Ahles T, et al. Cognitive and brain structural changes in a lung cancer population. J Thorac Oncol 2015;10:38-45.

6. Kaur S, Sharma R, Rastogi S. Demystifying the association of chemotherapy and cognition: How close are we? J Clin Oncol 2016. pii: JCO687400.

7. Wefel JS, Vidrine DJ, Marani SK, Swartz RJ, Veramonti TL, Meyers CA, et al. A prospective study of cognitive function in men with non-seminomatous germ cell tumors. Psychooncology 2014;23:626-33.

8. Jacola LM, Krull KR, Pui CH, Pei D, Cheng C, Reddick WE, et al. Longitudinal assessment of neurocognitive outcomes in survivors of childhood acute lymphoblastic leukemia treated on a contemporary chemotherapy protocol. J Clin Oncol 2016;34:1239-47.

9. Inagaki M, Yoshikawa E, Matsuoka Y, Sugawara Y, Nakano T, Akechi $\mathrm{T}$, et al. Smaller regional volumes of brain gray and white matter demonstrated in breast cancer survivors exposed to adjuvant chemotherapy. Cancer 2007;109:146-56.

10. Jenkins V, Thwaites R, Cercignani M, Sacre S, Harrison N, Whiteley-Jones $\mathrm{H}$, et al. A feasibility study exploring the role of pre-operative assessment when examining the mechanism of 'chemo-brain' in breast cancer patients. Springerplus 2016;5:390.

11. Menning S, de Ruiter MB, Veltman DJ, Boogerd W, Oldenburg HS, Reneman L, et al. Changes in brain activation in breast cancer patients depend on cognitive domain and treatment type. PLoS One 2017;12:e171724.

12. Bruno J, Hosseini SM, Kesler S. Altered resting state functional brain network topology in chemotherapy-treated breast cancer survivors. Neurobiol Dis 2012;48:329-38.

13. Moore HC, Parsons MW, Yue GH, Rybicki LA, Siemionow W. Electroencephalogram power changes as a correlate of chemotherapy-associated fatigue and cognitive dysfunction. Support Care Cancer 2014;22:2127-31.

14. Junghöfer $M$, Elbert $T$, Leiderer $P$, Berg $P$, Rockstroh $B$. Mapping EEG-potentials on the surface of the brain: A strategy for uncovering cortical sources. Brain Topogr 1997;9:203-17.

15. Robinson AK, Venkatesh $\mathrm{P}$, Boring MJ, Tarr MJ, Grover $\mathrm{P}$, Behrmann $\mathrm{M}$, et al. Very high density EEG elucidates spatiotemporal aspects of early visual processing. Sci Rep 2017;7:16248.

16. Brodbeck V, Spinelli L, Lascano AM, Wissmeier M, Vargas MI, Vulliemoz $\mathrm{S}$, et al. Electroencephalographic source imaging: A prospective study of 152 operated epileptic patients. Brain 2011;134:2887-97.

17. Lindenbeg A. From maps to mechanisms through neuroimaging of schizophrenia. Nature 2010;468:194.

18. Mathan S, Smart A, Ververs T, Feuerstein M. Towards an index of cognitive efficacy EEG-based estimation of cognitive load among individuals experiencing cancer-related cognitive decline. Conf Proc IEEE Eng Med Biol Soc 2010;2010:6595-8.

19. Alvarez J, Meyer FL, Granoff DL, Lundy A. The effect of EEG biofeedback on reducing postcancer cognitive impairment. Integr Cancer Ther 2013;12:475-87.

This is an open access journal, and articles are distributed under the terms of the Creative Commons Attribution-Non Commercial-ShareAlike 4.0 License, which allows others to remix, tweak, and build upon the work non-commercially, as long as appropriate credit is given and the new creations are licensed under the identical terms

\begin{tabular}{|l|l|}
\hline \multicolumn{2}{|c|}{ Access this article online } \\
\hline Quick Response Code: & Website: \\
& www.ijmpo.org \\
& DOI: \\
\hline
\end{tabular}

How to cite this article: Rastogi S, Sharma R, Kaur S. Cognitive studies for cancer survivors in India: Is this the right time or should we cross the bridge only when we will come to it?. Indian J Med Paediatr Oncol 2018;39:269-71. 